\title{
A new way to see a broncho-pleural fistula
}

Elaine Soon ${ }^{1,2} \mathrm{PhD}$, Pasupathy Sivasothy ${ }^{2}$ FRCP.

${ }^{1}$ Dept. of Medicine, Box 157, Level 5, Addenbrookes' Hospital, Cambridge CB2 0QQ, UK.

${ }^{2}$ Interventional Pulmonology Service, Cambridge University Hospitals NHS Foundation Trust, Cambridge CB2 0QQ, UK.

Correspondence to:

Dr P Sivasothy,

Interventional Pulmonology Service

Box 40, Cambridge University Hospitals NHS Foundation Trust,

Hills Road, Cambridge CB2 0QQ,

United Kingdom.

Tel: +44 1223216645

Fax: +44 1223216953

E-mail: pasupathy.sivasothy@addenbrookes.nhs.uk

All authors took part in the writing and approval of this manuscript.

The authors have no relevant disclosures or conflicts of interests.

Funding sources: not applicable.

The patient has given consent for publication.

Short running head: Broncho-pleural fistula

Descriptor number: 9.24 Interventional Pulmonology/Bronchoscopy

Total word count: 518

These images have never been previously published. 
A 77-year old man presented with five episodes of sepsis to different hospitals in the year after having a left lower lobectomy for squamous cell carcinoma. In the first two episodes a rim-enhancing left pleural effusion was seen and drained under computed tomography guidance. In his third and subsequent episodes the effusion was noted as being present but was considered too small and loculated to drain effectively. His admission chest radiograph at his fifth presentation, which shows non-specific changes at the left base, is shown in Fig.1A. Computed-tomography and bronchoscopy failed to demonstrate a broncho-pleural fistula. Therefore we combined both techniques. The patient was bronchoscoped in a near upright position under conscious sedation with midazolam. At bronchoscopy, 15mls of Niopam 300 (an iodine-containing contrast agent routinely used in computer tomography enhancement, angiography and arthrography) was diluted with an equal volume of sterile normal saline to reduce its viscosity. Three $10 \mathrm{ml}$ aliquots of diluted Niopam 300 were gently injected by hand over five minutes into the left lower bronchial stump under fluoroscopic guidance (Fig.1B). Contrast was seen pooling passively in a cavity as well as extending in an arboreal pattern into the bronchi (Fig.1C). Subsequent broncho-alveolar lavage was performed with $120 \mathrm{mls}$ of sterile normal saline and aspirated to dryness. Fluoroscopy demonstrated residual contrast in a pleural-based cavity (Fig.1D) while the arboreal pattern was lost; indicating washout of contrast from the airways. The patient was observed in the bronchoscopy recovery area for two hours and then sent to the radiology suite. Finally computed-tomography postbronchoscopy provided definitive proof of a fistula (Fig.1E).

Broncho-pleural fistula is a significant complication affecting $~ 2 \%$ of post-resection patients $^{1}$. There are no established guidelines for their management and most clinicians focus on intensive antibiotic and nutritional support, empyema drainage and closure of the tract by surgical or bronchoscopic means ${ }^{2}$. Many patients are debilitated due to multiple factors such 
as surgery, repeated bouts of sepsis prior to recognition of the diagnosis and pre-existing comorbidities and hence may not be suitable for further surgical intervention. Novel interventions include fistula occlusion with fibrin sealant ${ }^{2}$, other sclerosants ${ }^{3}$ or endobronchial valves/coils ${ }^{4,5}$. We describe a novel method of visualising small bronchopleural fistulae which cannot be detected with conventional bronchoscopy or imaging. Our method requires access to bronchoscopy services, contrast media, and fluoroscopy; which are usually available in most district general hospitals. Proving the existence of a fistula then leads to the possibility of tract closure to end the vicious cycle of episodes of sepsis. We note that this method is dependent on the availability of fluoroscopy in the endoscopy suite. An alternative method in patients with indwelling pleural drains would be to inject methylene blue into the bronchial stump during bronchoscopy in a manner similar to previously described. The appearance of methylene blue in the drain output then proves the existence of a fistula. Our patient was deemed too frail for surgery and is receiving antibiotics pending bronchoscopic glue injection. We recommend that clinicians maintain a high index of suspicion and be open to using novel approaches such as the one we describe if a bronchopleural fistula cannot be localised using conventional methods. 


\section{References}

1. Fuso L, Varone F, Nachira D, Leli I, Salimbene I, Congedo MT, Margaritora S, Granone P. Incidence and Management of Post-Lobectomy and Pneumonectomy Bronchopleural Fistula. Lung. 2016 Jan 11 PMID: 2675356

2. Shrestha P, Safdar SA, Jawad SA, Shaaban H, Dieguez J, Elberaqdar E, Rai S, Adelman M. Successful closure of a bronchopleural fistula by intrapleural administration of fibrin sealant: a case report with review of literature. $N$ Am J Med Sci. 2014; 6:487-90.

3. Cardillo G, Carbone L, Carleo F, Galluccio G, Di Martino M, Giunti R, Lucantoni G, Battistoni P, Batzella S, Dello Iacono R, Petrella L, Dusmet M. The Rationale for Treatment of Postresectional Bronchopleural Fistula: Analysis of 52 Patients. Ann Thorac Surg. 2015; 100:251-257.

4. Podgaetz E, Andrade RS, Zamora F, Gibson H, Dincer HE. Endobronchial Treatment of Bronchopleural Fistulas by Using Intrabronchial Valve System: A Case Series. Semin Thorac Cardiovasc Surg. 2015;27:218-222.

5. Katoch CD, Chandran VM, Bhattacharyya D, Barthwal MS. Closure of bronchopleural fistula by interventional bronchoscopy using sealants and endobronchial devices. Med J Armed Forces India 2013;69:326-329. 
A

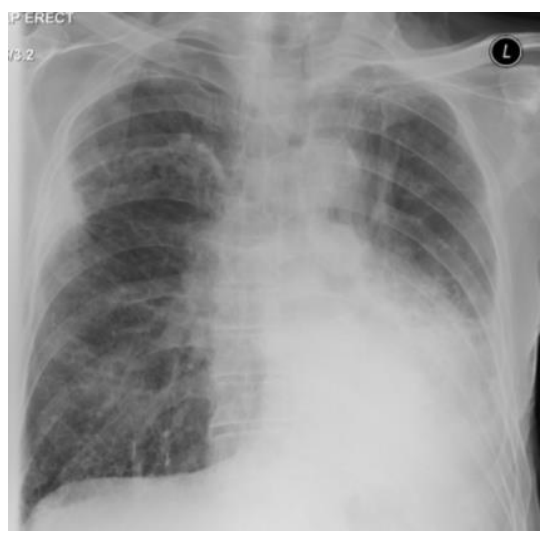

B

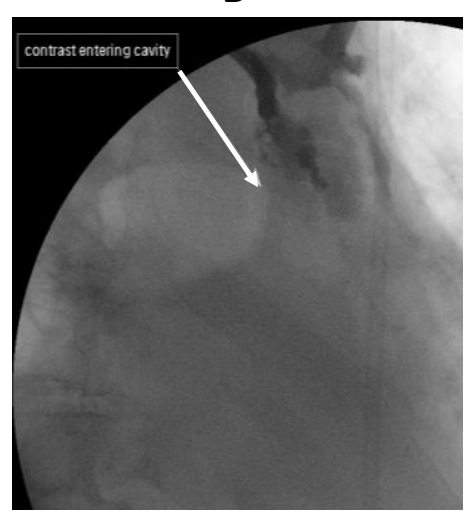

E

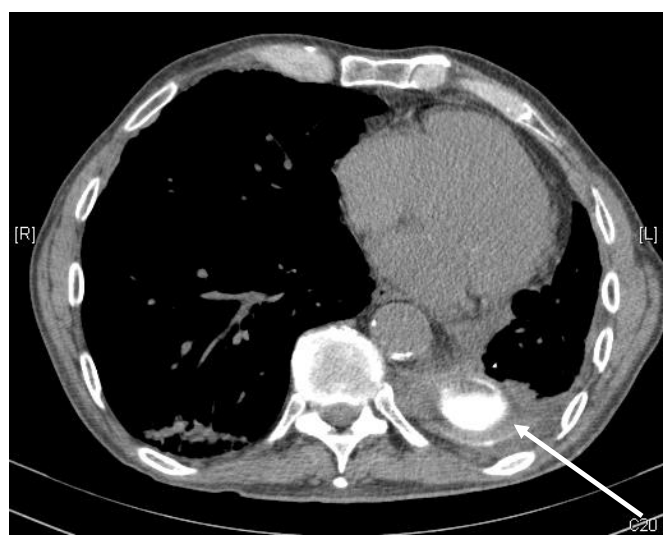

C

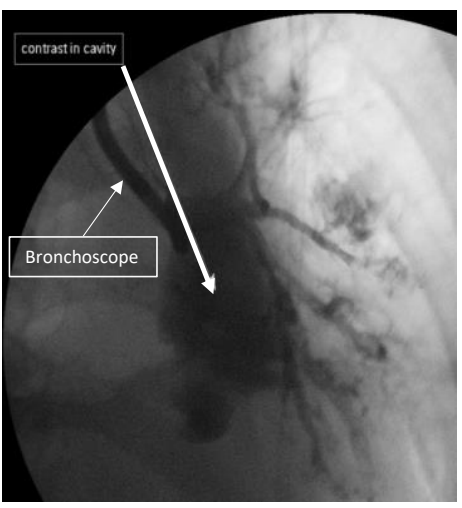

D

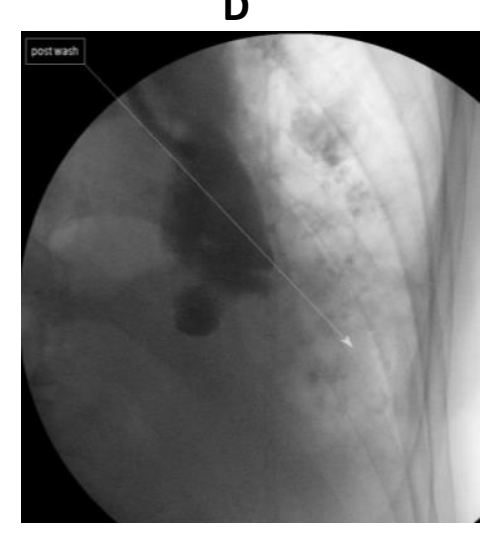

\section{Figure 1}

(A) Plain chest radiograph of the patient on admission, showing non-specific changes at the left base.

(B) Fluoroscopic image showing moment of contrast injection into left bronchial stump. The thick white arrow points at contrast entering the pleural cavity.

(C) Fluoroscopic image showing both contrast in the pleural cavity (thick white arrow) and also in the airways in an arboreal pattern. The bronchoscope is indicated by a thin white arrow.

(D) Fluoroscopic image showing persistence of contrast in a pleural cavity after bronchoalveolar lavage while the arboreal pattern is lost (thin white arrow shows 'wash out' from airways) 
(E) Computed tomography image post-bronchoscopy with patient lying supine shows dependent pooling of contrast in a pleural-based cavity (thick white arrow). 